\title{
Desarrollo de la biomasa y raíz en plantas de lulo (Solanum quitoense var. septentrionale) en respuesta al sombrío y anegamiento
}

\section{Biomass and root development response of lulo (Solanum quitoense var. septentrionale) plants to shading and waterlogging}
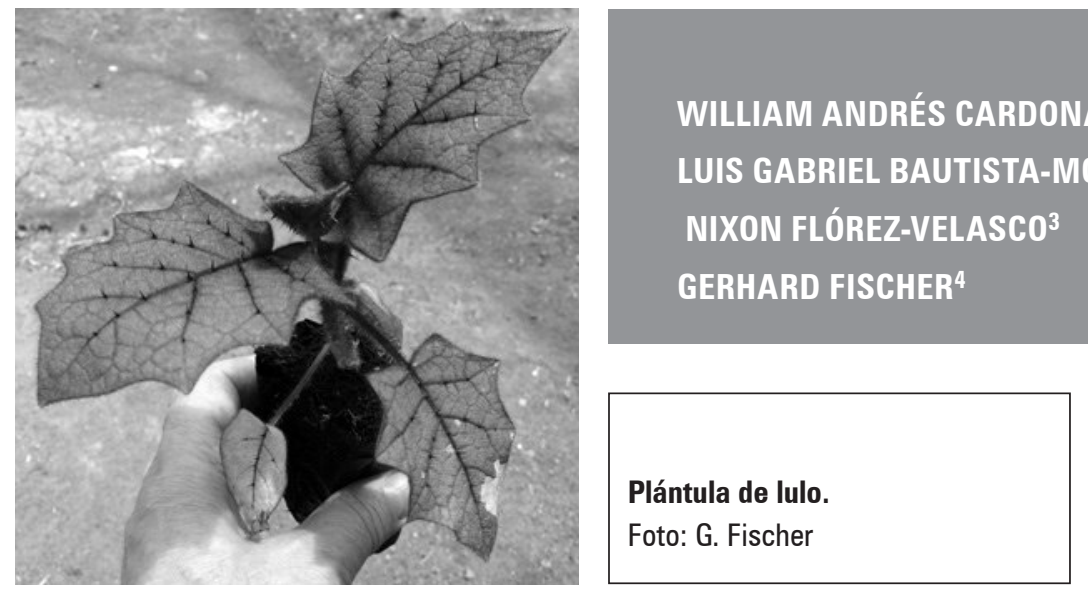

\section{RESUMEN}

El cambio y la variabilidad climática incrementan los periodos de lluvias, generando inundaciones y/o anegamientos, los cuales impactan en el desarrollo fisiológico de las especies cultivadas. En lulo, aunque se recomienda su siembra bajo penumbra, se estudió el efecto del estrés por anegamiento en condiciones de sombrío bajo invernadero. Las plantas de lulo, de 10 semanas de edad, fueron sembradas en materas de plástico de $5 \mathrm{~L}$ con un sustrato suelo y arena cuarcítica en proporción 1:1 v/v, se determinó el efecto de $65 \%$ sombrío en los cuatro periodos del anegamiento de 0,3, 6 y 9 días, más 9 días de recuperación c/u, sobre el desarrollo de la biomasa de planta y las raíces. El sombrío disminuyó la acumulación de biomasa en todos los órganos vegetativos, especialmente en las hojas (-22,7\%). No se presentaron interacciones entre el sombrío y el anegamiento. La raíz resultó ser el órgano más afectado por los periodos de mayor anegamiento ( 6 y 9 días), con reducciones del diámetro del cuello radical, longitud de raíz pivotante y del volumen y peso seco de raíz, mientras que, en consecuencia, la relación de biomasa entre la parte aérea y la raíz se aumentó. Durante el periodo de recuperación de 9 días persistió el efecto negativo de la privación del oxígeno en la rizósfera y, más bien, se agravó todavía más para los variables volumen y longitud de la raíz.

Facultad de Ciencias Agrarias, Programa de Maestría en Ciencias Agrarias, Línea de Investigación en Suelos y Aguas, Universidad Nacional de Colombia, Bogotá (Colombia).

2 Facultad de Ciencias Agrarias, Programa de Maestría en Ciencias Agrarias, Línea de Investigación en Fitopatología, Universidad Nacional de Colombia, Bogotá (Colombia).

3 Facultad de Ciencias Agrarias, Programa de Maestría en Ciencias Agrarias, Línea de Investigación en Fisiología de Cultivos, Universidad Nacional de Colombia, Bogotá (Colombia).

4 Facultad de Ciencias Agrarias, Departamento de Agronomía, Universidad Nacional de Colombia, Bogotá (Colombia).

5 Autor para correspondencia.wacardona@unal.edu.co 


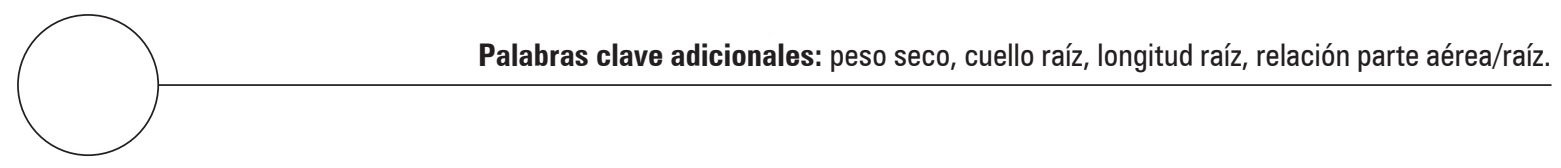

\section{ABSTRACT}

Climate change and its variability increase rainy periods, generating flooding and waterlogging, which affect the physiological development of cultivated species. In lulo, although growth under shade is recommended, the effect of stress due to waterlogging was studied under conditions of partial shading in greenhouse. Young lulo plants were planted in $5 \mathrm{~L}$ plastic pots with soil and sand quartzite at a ratio of $1: 1 \mathrm{v} / \mathrm{v}$ as substrate. The effect of $65 \%$ shading and no shading during four periods of waterlogging, 0, 3, 6 and 9 days, and a recovery period of 9 days each on the development of plant biomass and roots was determined. The shading decreased biomass accumulation in all of the vegetative organs, especially the leaves $(-22.7 \%)$. No interactions between the shading and waterlogging were measured. The root system proved to be the organ most affected by the periods of increased waterlogging (over 6 and 9 days), with reductions in the diameter of the root collar, length of taproot, volume and dry weight of roots, while; as a consequence, the shoot/root ratio of the biomass increased due to rhizosphere oxygen deprivation. During the recovery period of 9 days, the negative effect of the waterlogging persisted and was further aggravated for the volume and root length variables.

Additional keywords: dry weight, root collar, root length, shoot/root ratio.

El lulo (Solanum quitoense Lam.) es un arbusto tropical originario de los bosques húmedos de los Andes de Suramérica (Messinger y Lauerer, 2015), incluyendo a Colombia, Ecuador y Perú dentro del centro primario de su diversidad genética (Bonet y Cárdenas, 2012). Las dos variedades botánicas de lulo más importantes en Colombia son la septentrionale y la quitoense. La primera se caracteriza por la presencia de espinas en el tallo y en las hojas y por una mayor acidez de sus frutos, mientras la segunda variedad no presenta espinas y sus frutos son más dulces (Morton, 1987).

En Colombia y Ecuador es considerado como un producto importante en la canasta familiar y cuenta con un alto potencial agroindustrial en la obtención de pulpa, jugos, néctares y con- centrados (González et al., 2014; Flórez et al., 2008). En 2014, la superficie sembrada en Colombia con este frutal fue de 9.829 ha, de las cuales se obtuvieron 80.313 t y un rendimiento promedio de 9,54 $\mathrm{t} \mathrm{ha}^{-1}$, a partir de $8.418 \mathrm{ha}$ cosechadas y los departamentos de Huila, Valle del Cauca, Antioquia, Santander, Boyacá y Caldas son los de mayor participación a nivel nacional (MADR, 2014).

Por ser una planta originaria de los bosques húmedos, presenta buena adaptación a regiones frescas y sombreadas, se presume que es una planta de semipenumbra, con ruta fotosintética C3 (Medina et al., 2006). Por ello, se recomienda su siembra bajo penumbra natural o artificial (Bonet y Cárdenas, 2012). Sin embargo, como producto de la domesticación, se han establecido 
cultivos comerciales en ambientes de alta iluminación y libre exposición, en especial en los materiales sin espinas (Medina et al., 2006).

El Panel Intergubernamental de Cambio Climático reporta que el incremento en las precipitaciones, causadas principalmente por el calentamiento global resultado de las actividades humanas, tendrá un mayor impacto en las regiones tropicales como consecuencia del aumento del ciclo hidrológico mundial (Bailey-Serres y Voeseneck, 2008). En Colombia durante los años 2010 y 2011, el incremento de las precipitaciones, causadas por el fenómeno de La Niña, afectó seriamente las regiones Caribe y Andina del territorio; en dicha temporada, se registró la afectación del 87,5\% de los departamentos del país (DANE, 2011).

Los periodos de anegamiento en periodos de alta pluviosidad o riego excesivo, unidos a un pobre drenaje del suelo (Pardos, 2004; Torres y Fischer, 2016) dificultan el desarrollo radical y la fisiología de las especies cultivadas, generando grandes pérdidas de producción agrícola en todo el mundo (Bailey-Serres et al., 2012). Anegamiento e inundación conllevan a una saturación de los poros en el suelo con agua que normalmente están ocupados con aire (Lambers et al., 2008). En consecuencia, en el suelo se presenta la reducción del oxígeno $\left(\mathrm{O}_{2}\right)$ libre a cambio de la acumulación de dióxido de carbono $\left(\mathrm{CO}_{2}\right)$, que afecta el potencial redox del suelo, cambiando la disponibilidad de nutrientes como manganeso $\left(\mathrm{Mn}^{4+}\right)$, hierro $\left(\mathrm{Fe}^{3+}\right)$ y sulfato $\left(\mathrm{SO}_{2}{ }^{4-}\right)$ e incrementando la solubilidad de metales tóxicos (Zeng et al., 2013). Adicionalmente aumenta la producción de sustancias tóxicas como etanol, acetaldehído, compuestos cianógenos y etileno (Kozlowski, 1997). Por el lavado de las raíces se presenta una pérdida de nutrientes minerales como compuestos solubles de nitrógeno originando un desequilibrio nutricional debido a la actividad anaeróbica microbiana (Pardos, 2004).

La poca disponibilidad de $\mathrm{O}_{2}$ en el suelo anegado reduce la difusión del oxígeno disuelto el cual solo queda disponible en una capa delgada ubicada cerca de la superficie (Taiz y Zeiger, 2010). Esta condición de hipoxia se ha convertido en un estrés abiótico importante que afecta el crecimiento, desarrollo, productividad e incluso la distribución de las especies (Kozlowski, 1997) y que muestra un fuerte efecto negativo sobre el crecimiento y la actividad fisiológica de las raíces (Pallardy, 2007).

La mayoría de las especies vegetales son susceptibles, a un mayor o menor grado, a esta adversidad y han desarrollado adaptaciones a estas condiciones anaeróbicas (Bailey-Serres y Voesenek, 2008). Las plantas pueden llegar a tolerar el encharcamiento mediante mecanismos que implican el transporte y suministro de oxígeno a los órganos sumergidos, dentro de estos la formación de aerénquima y la inserción de raíces adventicias, cerca de la superficie del agua, son los más importantes (Moreno y Fischer, 2014). El aerénquima es un conjunto de canales que admite la difusión interna del oxígeno atmosférico y el proveniente de la fotosíntesis de las partes aéreas hacia las raíces anegadas favoreciendo la respiración aeróbica de este órgano vital para la planta (Yamauchi et al., 2013).

La radiación solar es la fuente energética más importante para la asimilación del carbono y el crecimiento de la planta (Valladares y Niinemets, 2008), por lo cual cualquier reducción en esta causará efectos sobre la fotosíntesis y la tasa de crecimiento (Mielke y Schaffer, 2010).

Las plantas están frecuentemente limitadas por múltiples factores de estrés que operan de forma simultánea, en este sentido, las respuestas fisiológicas a efectos separados de anegamiento y sombrío sugieren que ambos factores interactúan de tal forma, que el efecto adverso del sombrío se amplificará en suelos anegados (Laan et al., 1990). La interacción de estas dos condiciones puede afectar el crecimiento de plantas de forma independiente o interactuar haciendo que un factor reduzca y/o incremente el impac- 
to de factores adicionales (Baracaldo et al., 2014). Mommer et al. (2005) constataron que existen fuertes similitudes entre las respuestas de aclimatización a sombrío e inmersión en agua de las plantas.

Dada la importancia del cultivo de lulo para el sector frutícola colombiano y el poco conocimiento que existe en el estudio de la fisiología de esta especie en condiciones de variabilidad climática, así como la falta del entendimiento sobre los mecanismos como las plantas reaccionan a un suelo saturado de agua (Voesenek et al., 2014), el presente estudio tuvo como objetivo determinar los efectos que causan el sombrío y el anegamiento sobre la formación de biomasa de los órganos de la planta y los componentes del desarrollo radical.

\section{MATERIALES Y MÉTODOS}

\section{Material vegetal y condiciones del experimento}

El experimento se llevó a cabo en un invernadero de cubierta plástica de la Facultad de Ciencias Agrarias de la Universidad Nacional de Colombia, sede Bogotá, localizado a $4^{\circ} 35^{\prime} 56^{\prime \prime} \mathrm{N}$ y $74^{\circ} 04^{\prime} 51^{\prime \prime} \mathrm{W}$, con altitud de $2.556 \mathrm{~m}$. El material vegetal utilizado consistió en plantas de lulo de 10 semanas de edad, propagadas por semilla. Cada planta fue trasplantada en una matera de plástico de $5 \mathrm{~L}$ de capacidad, que contenía como sustrato suelo tamizado y arena cuarcítica en proporción 1:1 v/v. Las propiedades físicas y químicas del sustrato empleado fueron: $\mathrm{pH} 5,2 ; \mathrm{CO} 6,33 \%$; 0,55\%; Ca 6,21; K 1,79; Mg 1,42 y Na 0,29 meq $100 \mathrm{~g}^{-1}$; P 37,2; Cu 0,20; Fe 47; Mn 4,57; Zn 1,45 y B $0,34 \mathrm{mg} \mathrm{kg}^{-1}$; AI 0,23 meq $100 \mathrm{~g}^{-1}$; CICE 9,94 meq $100 \mathrm{~g}^{-1}$; con textura franco arenosa.

Para el primer factor de estrés, el sombrío, los dos niveles fueron: (1) sin sombrío (SS) adicional, exponiendo las plantas a la radiación solar bajo la cubierta plástica del invernadero $(66.69 \pm 1.1 \mathrm{~lx})$ y (2) con sombrío (CS), colocando las plantas en una cámara cubierta por todos los lados con polisombra negra, instalada a 1,5 m por encima de las plantas, que brindó un porcentaje de sombra del $65 \pm 4 \%$, con una radiación de $29.94 \pm 3.4$ lx.

Para el factor de anegamiento se probaron cuatro niveles correspondientes a diferentes tiempos de duración así: 0, 3, 6 y 9 d, con un tiempo de recuperación de 9 d. Para ello se cerraron herméticamente las aberturas de drenaje de las materas y mediante la adición de agua de riego se mantuvo permanentemente una lámina de $3 \mathrm{~cm}$ por encima del nivel del sustrato, durante los días de duración de cada tratamiento. El estrés por anegamiento se suspendió destapando los orificios de drenaje de las materas en los días antes mencionados.

Las condiciones de temperatura del aire fueron similares para las dos condiciones de sombrío, CS y SS; con 25,3 y $25,6^{\circ} \mathrm{C}$, respectivamente, y la humedad relativa al interior de la cámara de polisombra fue superior con respecto al área sin sombrío, registrando valores de $61,2 \%$ y $25,7 \%$, respectivamente.

\section{Parámetros del desarrollo radical}

Se registró el diámetro del cuello de raíz empleando un calibrador Vernier digital 8 in, referencia 3416. Para determinar la longitud de la raíz pivotante se midió la longitud de estas mediante una cinta métrica, mientras que el volumen se calculó mediante un cilindro graduado (probeta) que contenía un volumen conocido de agua (250 $\mathrm{mL}$ ), del cual se registró el volumen desplazado al sumergir las raíces evaluadas.

\section{Biomasa seca de los órganos}

Se separaron las plantas en hojas, tallo y raíz, secando las muestras en un horno universal a $80^{\circ} \mathrm{C}$ durante $48 \mathrm{~h}$, hasta peso constante, y posteriormente se registró el peso seco de las biomasas empleando una balanza de precisión. 


\section{Relación entre la biomasa aérea/radical}

Con los pesos obtenidos a partir de las estructuras separadas, se calculó la relación parte aérea/ radical, esto mediante el cociente entre el peso seco de la parte aérea y el peso seco de la raíz.

\section{Diseño experimental y análisis estadístico de los datos}

Se utilizó un diseño completamente aleatorizado con arreglo factorial. El primer factor corresponde a los periodos de anegamiento y recuperación y el segundo factor a sombrío, con tres repeticiones por tratamiento, donde cada una consistió de una planta individual en matera. Se comprobaron los supuestos mediante test de Levene y Shapiro-Wilk. Posteriormente, los datos se analizaron mediante ANAVA de dos vías. La prueba Tukey se utilizó para determinar el efecto de los tratamientos sobre las variables. Los datos fueron analizados usando Statistix v 9.0 (Analytical Software,Tallahassee, FL).

\section{RESULTADOS Y DISCUSIÓN}

\section{Sombrío}

El análisis de varianza arrojó diferencias significativas en la reducción de la biomasa seca total y de los órganos de la planta, así mismo como el diámetro del tallo basal, debido al 65\% sombrío, mientras las otras variables no fueron afectadas por el factor luz (tablas 1 y 2). Este resultado refleja la importancia de la luz en la producción de biomasa a través de la fotosíntesis y la posterior translocación de los fotoasimilados (Taiz y Zeiger, 2010; Fischer et al., 2012), lo que también concuerda con lo encontrado por Casierra-Posada et al. (2013) en plantas de lulo sombreados que redujeron en un $27,5 \%$ la tasa de asimilación neta (TAN).

Resultados similares observaron Mielke y Schaffer (2010) en el arbusto suramericano Eugenia uniflora que desarrolló mayores pesos secos de la parte aérea, raíz y peso seco total de 30\%, 50\% y $33 \%$ en plantas que recibieron plena luz, comparado con plantas bajo luz parcial (40,5 y 10,7 mol m${ }^{-2}$ día $^{-1}$ de densidad del flujo fotosintético, respectivamente). Del mismo modo, CasierraPosada et al. (2013) registraron en lulo una reducción del $27,3 \%$ en el peso seco total acumulado por las plantas que estuvieron cubiertas con una polisombra.

Es para considerar que aunque el sombrío fue del $65 \%$, la biomasa total se redujo solamente en un 21\%, mostrando la adaptación del lulo a ambientes de penumbra (Bonet y Cárdenas, 2012), pero, al mismo tiempo, confirmando sus altos rendi-

Tabla 1. Resumen de análisis de varianza del efecto de los tratamientos de sombrío y anegamiento sobre variables de crecimiento en plantas de lulo.

\begin{tabular}{|c|c|c|c|c|}
\hline & \multirow{2}{*}{ Abrev. } & \multicolumn{3}{|c|}{ Fuente de variación } \\
\hline & & Sombrío & Anegamiento & Sombrío x Anegamiento \\
\hline Peso seco hoja & PSH & $* *$ & ns & ns \\
\hline Peso seco tallo & PST & * & $* * *$ & ns \\
\hline Peso seco raíz & PSR & * & *** & ns \\
\hline Peso seco total & PSTL & $* *$ & ns & ns \\
\hline Relación parte aérea raíz & $\mathrm{A} / \mathrm{R}$ & ns & $* * *$ & ns \\
\hline Volumen raíz & VR & ns & $* * *$ & ns \\
\hline Longitud raíz & $\mathrm{LR}$ & $\mathrm{ns}$ & $* * *$ & ns \\
\hline Diámetro tallo & DT & ** & *** & ns \\
\hline
\end{tabular}

${ }^{*},{ }^{* *} y * *$ diferencias significativas en el nivel de probabilidad de 0,$05 ; 0,01$ y 0,001 , respectivamente. NS, no significativo en $\alpha=0,05$. 
mientos en ambientes de elevada luminosidad (Medina, 2006), lo que expresa la buena plasticidad fisiológica de esta planta a la disponibilidad de luz (Lavinski et al., 2007) y, además, no se pudo confirmar lo reportado por Morton (1987) que el lulo no es adaptado a una plena luz solar. Sin embargo, en este contexto, vale mencionar que expresó Lobo (2000) que la plena exposición solar redujera el periodo productivo de la planta de lulo debido a una acelerada actividad metabólica.

La reducción del diámetro del tallo basal por la sombra está directamente relacionada con la menor acumulación de biomasa en este órgano (tabla 2), además, podría ser una consecuencia de menores tasas de translocación de carbohidratos hacía las raíces, necesitando un sistema vascular de menor diámetro, mientras en plantas no estresadas lumínicamente el tallo y las raíces reciben cantidades suficientes de carbohidratos, especialmente en la fase inicial del crecimiento de la planta (Fischer et al., 2012). Hansen (1978) reporta tasas de flujo de fotoasimilados más altas en doseles de plantas de manzano a plena luz comparado con plantas sometidas a deficiente luz. También Dwivedi y Dwivedi (2012) resaltan que los cambios inducidos por baja intensidad lumínica pueden conllevar a un reducido flujo de carbohidratos hacía las raíces, interviniendo negativamente en la respiración y la actividad enzimática de este órgano y, así disturbando, por lo menos en parte, el metabolismo de toda la planta.

Como indica la tabla 1, entre sombrío y anegamiento no se registraron interacciones significa- tivas $(P>0,05)$. Tampoco Baracaldo et al. (2014) encontraron interacciones entre el sombrío de $56 \%$ y el anegamiento en tomates chonto durante 4 , 8 y $12 \mathrm{~d}$, sin embargo, con un enfoque funcional (usando el modelo de Gompertz), estos autores vieron una reducción de la biomasa seca total de las plantas sombreadas y anegadas durante $12 \mathrm{~d}$, a partir del día 20 del inicio del anegamiento, comparado con los demás tratamientos.

\section{Anegamiento}

El factor anegamiento mostró diferencias significativas $(P \leq 0,001)$ en seis de las ocho variables evaluadas (tabla 1), pero en el peso seco foliar que representa la mayor parte de la biomasa de la planta en la fase vegetativa (Medina et al., 2006) y así, consecuentemente, en la biomasa total, la falta de oxígeno en la rizósfera no produjo efectos marcados (tabla 1).

\section{Diámetro del tallo basal}

Con respecto al diámetro del cuello radical no hubo diferencias estadísticas en el desarrollo del grosor de este en las plantas no anegadas (control) durante los 9 d del estudio, sin embargo, las anegadas durante 6 y $9 \mathrm{~d}$ formaron tallos basales más gruesos $(P \leq 0,05)$ comparado con las del control o solamente anegadas por $3 \mathrm{~d}$ (figura 1A). También Flórez-Velasco et al. (2015) observaron en plantas de lulo anegadas un diámetro de tallo más grande que en las no anegadas, argumentando que esta respuesta se debe a la formación de tejido esponjoso (el aerénquima), el cual permite la difusión de gases, como el $\mathrm{O}_{2}$, del

Tabla 2. Efecto del sombrío sobre peso seco de la hoja (PSH), peso seco del tallo (PST), peso seco de la raíz (PSR), peso seco total (PSTL) y diámetro del tallo (DT) en plantas de lulo.

\begin{tabular}{|l|c|c|c|c|c|}
\hline \multicolumn{1}{r|}{ Tratamiento } & PSH $(\mathrm{g})$ & PST $(\mathrm{g})$ & PSR $(\mathrm{g})$ & PSTL $(\mathrm{g})$ & DT $(\mathrm{mm})$ \\
\hline Sin sombra & $3,00 \pm 0,16 \mathrm{a}$ & $2,18 \pm 0,13 \mathrm{a}$ & $1,39 \pm 0,12 \mathrm{a}$ & $6,60 \pm 0,33 \mathrm{a}$ & $8,78 \pm 0,31 \mathrm{a}$ \\
\hline Con sombra & $2,32 \pm 0,12 \mathrm{~b}$ & $1,81 \pm 0,10 \mathrm{~b}$ & $1,08 \pm 0,09 \mathrm{~b}$ & $5,24 \pm 0,26 \mathrm{~b}$ & $7,56 \pm 0,29 \mathrm{~b}$ \\
\hline CV $(\%)$ & 30,12 & 45,45 & 46,56 & 29,35 & 20,32 \\
\hline
\end{tabular}

Promedios con letras distintas indican diferencia significativa según la prueba de Tukey $(P \leq 0,05)$. Valores son promedios de tres repeticiones \pm error estándar. 
tallo aeróbico hacía las raíces hipóxicas/anóxicas en plantas anegadas e inundadas (Striker, 2012; Sairam et al., 2008).

Estos mismos resultados los obtuvieron NúñezElisea et al. (1999) y Mielke et al. (2005) en plantas de Annona glabra, sometidas a más de $10 \mathrm{~d}$ de anegamiento, en contrario a Aldana et al. (2014) quienes observaron a 50 d una reducción de $2 / 3$ del grosor del cuello radical en uchuva, anegadas durante $8 \mathrm{~d}$, en comparación con las plantas no estresadas. Del mismo modo, Fernández et al. (1997) observaron en la variedad de manzano 'Jonnee', injertadas sobre tres diferentes portainjertos, una reducción del aumento del área transversal del tronco, $5 \mathrm{~cm}$ por encima del injerto en plantas anegadas durante $32 \mathrm{~d}$, comparado con anegamientos más cortos.

Estos resultados, observados en las diferentes plantas estudiadas, confirman que la tolerancia al anegamiento depende mucho de la especie, además varía según la edad y fenología de la planta y la duración de las condiciones apóxicas/ anóxicas (Kozlowski, 1997).

El mayor crecimiento en grosor del tallo, debido al estrés por $9 \mathrm{~d}$ de anegamiento, se perdió significativamente $(P \leq 0,05)$ en la recuperación de las plantas en los $9 \mathrm{~d}$ siguientes (figura 1A), posiblemente porque la planta ya no requería continuar con el desarrollo del tejido esponjoso (Flórez-Velasco et al., 2015).

\section{Peso seco del tallo}

Esta variable no mostró diferencias estadísticas entre el control y las plantas anegadas en las tres fechas de evaluación (figura 1B). Se presentó una tendencia de aumento de biomasa entre los 3 y $6 \mathrm{~d}$ del ensayo, con una ligera reducción en su acumulación hacía los $9 \mathrm{~d}$. En las plantas de $3 \mathrm{~d}$ anegadas, su recuperación en los posteriores $9 \mathrm{~d}$ favoreció una mayor acumulación de masa seca $(P \leq 0,05)$ en el tallo, comparado con las plantas control (figura 1B). El efecto de la reducción de la biomasa del tallo, como lo registraron Baracaldo et al. (2014) en tomate, anegados 8 y 12 d y Aldana et al. (2014) en uchuva, anegadas 6 y 8 d, no pudo ser observado en las plantas de lulo.

\section{Peso seco de la raíz}

En la figura 3C se aprecia que las plantas sometidas a los periodos más largos de inundación (6 y 9 d) acumularon significativamente menor biomasa $(P \leq 0,05)$ en el sistema radical, en comparación con las plantas control. El efecto sobre la reducción de la biomasa radical fue tan grande, que las plantas no recuperaron en los $9 \mathrm{~d}$ posteriores (no anegadas) esta biomasa perdida (figura 3C).

Este resultado confirma que los daños más grandes en suelos anegados corresponden al sistema radical, debido a un suministro insuficiente de oxígeno (Yamauchi et al., 2013; Khondaker y Ozawa, 2007). La hipoxia o incluso la anoxia en la rizósfera es la consecuencia inmediata del anegamiento (Sauter, 2013), teniendo en cuenta que la resistencia a la difusión de oxígeno es hasta 10.000 veces mayor en soluciones del agua que en el aire (Vartapedian y Jackson, 1997).

Por otro lado, las concentraciones mínimas de oxígeno en el suelo pueden causar efectos tóxicos debido a la síntesis de sustancias como etanol y acetaldehído en la ruta de la respiración anaeróbica (Kozlowski, 1997). Como reportan Jiménez et al. (2012), en este proceso de la acidificación citosólica, se produce ácido láctico que disminuye el pH de las células induciendo la síntesis del acetaldehído.

Debido a la respiración anaeróbica de las raíces que produce solamente 2 ATP, en comparación con los 36 ATP en condiciones aeróbicas, la falta de energía afecta gravemente el metabolismo de las células radicales (Dwivedi y Dwivedi, 2012). López y Del Rosario (1983) observaron una disminución de la respiración radical del $72 \%$ en 

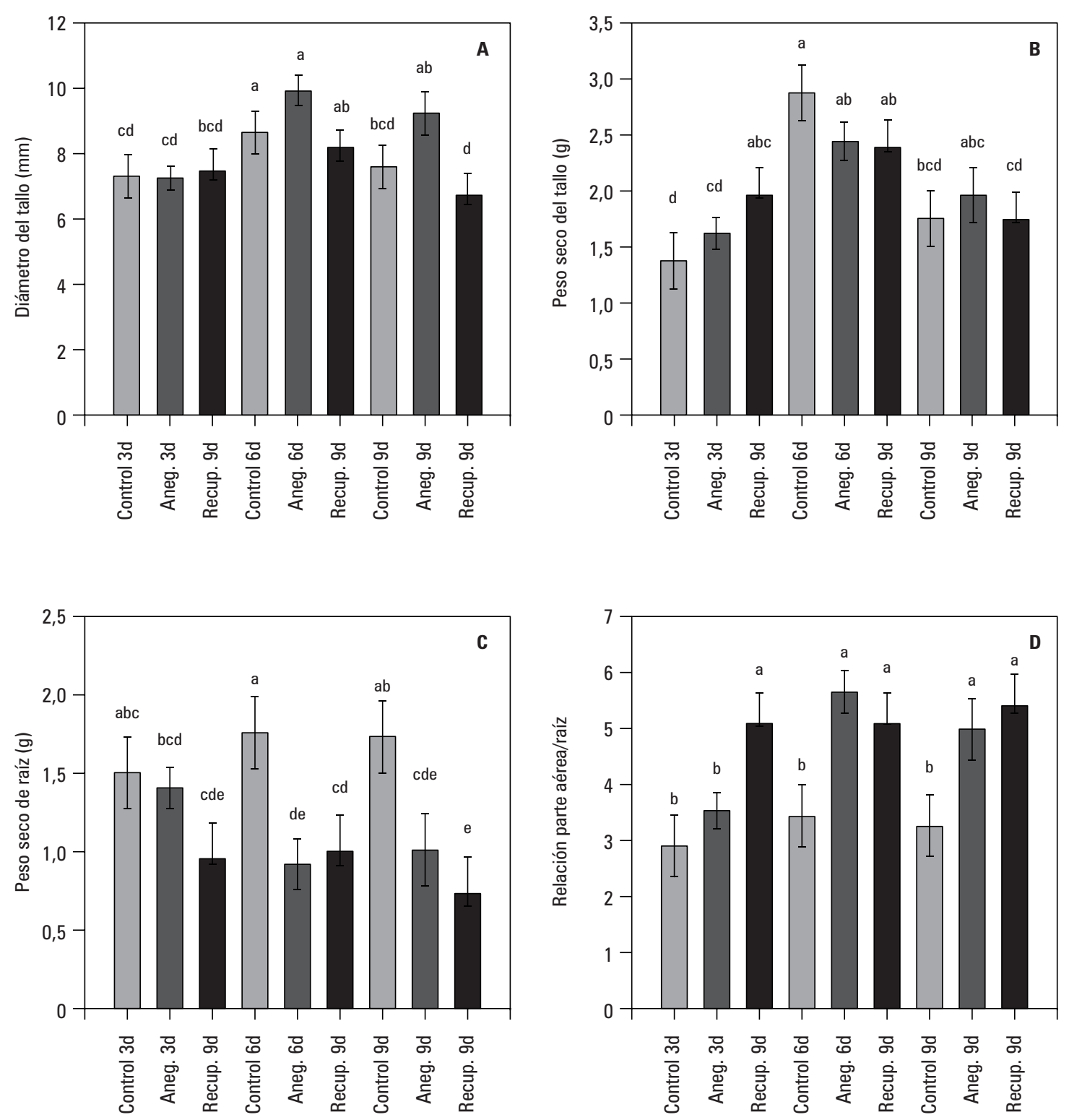

Figura 1. Efecto de tres periodos de anegamiento $(3,6$ y 9 días con 9 días de recuperación $\mathrm{c} / \mathrm{u})$ sobre $A)$ diámetro del tallo (corresponde al cuello radical), B) peso seco del tallo, C) peso seco de raíz y D) relación parte aérea/raíz en plantas de lulo. Valores son promedios de tres repeticiones \pm error estándar. Promedios con letras distintas indican diferencia significativa según la prueba de Tukey $(P \leq 0,05)$.

plantas de tomate anegadas por $10 \mathrm{~d}$. Steffens et al. (2005) concluyeron que la deficiencia de oxígeno en la rizósfera impide la síntesis de ATP, lo que conlleva a una muy baja toma de nutrientes, observado en el caso de trigo y avena.
Esta gran afectación del crecimiento radical por la hipoxia en la rizósfera coincide completamente con lo reportado por Larcher (2003) y lo encontrado por Fernández et al. (1997) en manzano por una disminución de la producción de nuevas 
raíces y por Baracaldo et al. (2014) en tomate y Aldana et al. (2015) en uchuva, en estas dos especies el anegamiento disminuyó ampliamente la acumulación de biomasa seca de las raíces comparado con los otros órganos vegetativos de la planta.

En todas las variables medidas en el presente estudio, la recuperación de las plantas, finalizando los $9 \mathrm{~d}$ del anegamiento, no obtuvo resultados positivos, lo que concuerda con los observado por Mielke y Schaffer (2010) en Eugenia uniflora, en la cual persistieron los efectos negativos de la hipoxia radical sobre la fotosíntesis y la acumulación de la biomasa.

\section{Peso seco total de la planta}

No se presentaron diferencias estadísticas $(P>0,05)$ por los tratamientos de anegamiento $(5,49$ g y 5,58 g en plantas anegadas y no anegadas, respectivamente), debido a que, sobre todo, la biomasa foliar (2,78 g y 2,69 g), la más abundante de la planta, tampoco fue influenciada significativamente $(P>0,05)$ por la hipoxia en el sistema radical (tabla 1 ).

Resultados similares encontraron Flórez-Velasco et al. (2015) en plantas de lulo anegadas, que tuvieron una nutrición nitrogenada baja (10 mg $\left.\mathrm{L}^{-1}\right)$, mientras con una alta (110 $\left.\mathrm{mg} \mathrm{L}^{-1}\right)$, estas plantas presentaron mayor tolerancia a la inundación y produjeron una biomasa total tres veces mayor que las con baja nutrición. Estos autores se refirieron por un lado, a una menor absorción de nitrógeno por las raíces y, por otro, midieron una menor eficiencia del fotosistema II (PSII) en las plantas anegadas con baja nutrición de $\mathrm{N}$. También Mielke y Schaffer (2010) registraron una reducción de biomasa total en Eugenia uniflora anegada en "solamente" $27 \%$, comparado con una masa seca menor de 58\% en las raíces.

\section{Relación biomasa parte aérea/raíz}

Debido al mayor crecimiento de la raíz al inicio del experimento, comparado con la parte aérea de la planta, las plantas anegadas mostraron a los $3 \mathrm{~d}$, una relación significativamente menor que las estresadas durante 6 y 9 d y esta relación fue también menor que en las plantas de recuperación después de los $3 \mathrm{~d}$ (figura 1D). También, por la afectación más grande de las raíces anegadas durante 6 y $9 \mathrm{~d}$ (figuras $1 \mathrm{C}$ y $2 \mathrm{~B}$ ), se presentó una alta relación parte aérea/raíz que superó significativamente la de las plantas control en estas dos evaluaciones (figura 1D).

Estos resultados coinciden con los encontrados en tomate (Baracaldo et al., 2014) y uchuva (Aldana et al., 2014) en los cuales se evidenció un fuerte aumento en la relación parte aérea/raíz cuando se incrementó la duración del anegamiento a partir de 8 y $6 \mathrm{~d}$, respectivamente, sin embargo, Flórez-Velasco et al. (2015) observaron en plantas de lulo que la relación de peso seco entre la parte aérea y raíz no varió por efecto del estrés por anegamiento.

La alta proporción de la parte aérea/raíz en las plantas anegadas durante 6 y $9 \mathrm{~d}$, que tampoco se disminuyó en los 9 d de recuperación, podría obedecer a la muerte del tejido radical por las condiciones de anoxia (Larcher, 2003) y del deficiente crecimiento de raíces nuevas (Moreno y Fischer, 2014), comparado con las plantas no anegadas. También Fischer y Orduz-Rodríguez (2012) afirman que en un suelo saturado de agua se mueren primero las raíces finas y fibrosas.

En los tejidos que sufren por las condiciones de hipoxia y todavía más por anoxia, Vartapetian y Jackson (1997), en su artículo de revisión, resumieron que las relaciones funcionales entre raíces y tallos están altamente disturbadas, y esto afecta gravemente la asimilación de carbohidratos y la utilización de los fotoasimilados de la planta.

\section{Longitud de raíz pivotante}

Esta variable no fue influenciada significativamente por los tratamientos del anegamiento, sin embargo el efecto negativo y significante 
se presentó después de los 9 d de recuperación, sobre todo, en las plantas de $9 \mathrm{~d}$ anegadas (figura 2A). También Mielke y Schaffer (2010) encontraron en Eugenia uniflora reducciones de crecimiento que se acentuaron en el periodo de recuperación del anegamiento, especialmente causado, en primer lugar, por una disminución de la fotosíntesis.

El exceso de agua en el suelo crea deficiencias de oxígeno en la zona de raíz, pudiéndose disminuir su crecimiento normal (Khondaker y Ozawa, 2007) y, además, la mayor producción de etileno en las condiciones hipóxicas disminuye la elongación de este órgano (Lambers et al., 2008). No se encontraron efectos más contundentes en esta variable, debido principalmente al hecho de que por tratarse de un muestreo destructivo, las raíces podrían haber sufrido un daño severo mecánico por la eliminación del suelo.

\section{Volumen de raíces}

Como muestran las figuras $2 \mathrm{~B}$ y 3 , el volumen de raíz fue altamente afectado en los periodos de anegamiento de 6 y $9 \mathrm{~d}$, con reducciones significativas $(P \leq 0,05)$ frente al control $(53 \%$ y $46 \%$, respectivamente). Este efecto negativo se aumentó $(P \leq 0,05)$ todavía más en el periodo de recuperación después de los 9 d (69\%), posiblemente por el afectado mecanismo de apertura de estomas en plantas sensibles a la hipoxia (Pallardy, 2007).

Se supone que la severa afectación del volumen radical podría haber sido causado, sobre todo, por una muy baja absorción de agua y nutrientes, generado por una reducida concentración del oxígeno libre en la rizósfera (Kozlowski, 1997) y un cierre estomático que, en consecuencia, generó una menor fotosíntesis y translocación de
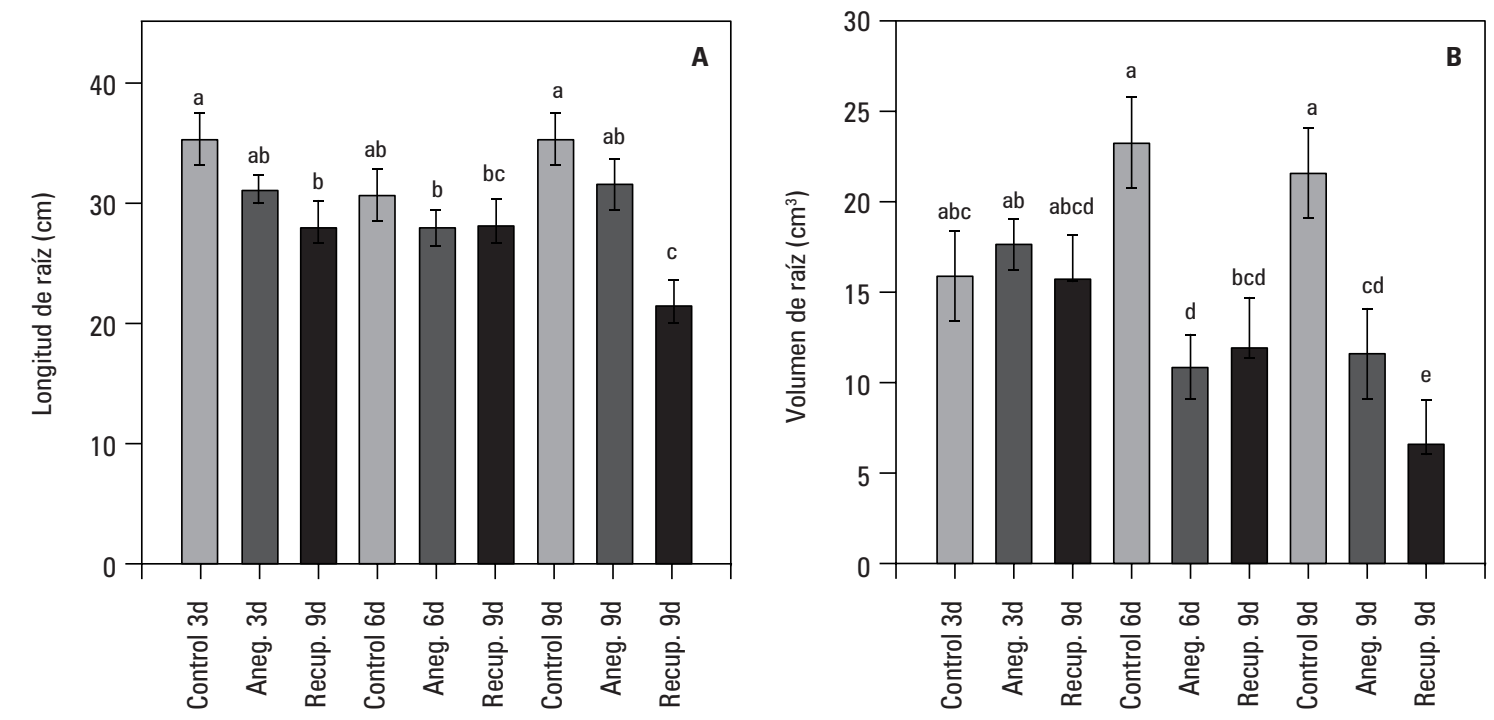

Figura 2. Efecto de tres periodos de anegamiento $(3,6$ y 9 días con 9 días de recuperación c/u) sobre $A)$ longitud de raíz y B) volumen de raíz en plantas de lulo. Valores son promedios de tres repeticiones \pm error estándar. Promedios con letras distintas indican diferencia significativa según la prueba de Tukey $(P \leq 0,05)$. 
carbohidratos (Najeeb et al., 2015; Taiz y Zeiger, 2010) afectando la formación de biomasa en las raíces (figura 3). Además, en raíces de un suelo anegado o inundado, la falta de oxígeno inhibe el transporte electrónico mitocondrial, la oxidación de NADH y la mayoría de las síntesis de ATP (Mielke y Schaffer, 2010) afectando el desarrollo de este órgano. También, Sauter (2013) reporta que en condiciones anaeróbicas o anóxicas las reservas de almidón en las raíces son gastadas en poco tiempo, haciendo difícil hasta imposible para la planta mantener el crecimiento y las funciones radicales.

Para especies y variedades que no están suficientemente adaptadas al estrés por anegamiento, autores como Yetisir et al. (2005) en sandía y Fernández et al. (1997) en manzano recomiendan el uso de patrones cuyo sistema radical tolere mejor las condiciones de hipoxia.

\section{CONCLUSIONES}

El sombrío del $65 \%$ de la luz incidente afectó la producción de biomasa en todos los órganos ve- getativos de la planta, especialmente en las hojas que son por naturaleza muy grandes en el lulo.

No se presentaron interacciones entre el sombrío y el anegamiento. El factor sombrío posiblemente no modifica los efectos de estrés por anegamiento en plantas de lulo en las condiciones de este experimento

El anegamiento tuvo grandes afectaciones sobre las raíces, siendo el órgano directamente perturbado por la falta de oxígeno en la rizósfera, disminuyendo la biomasa seca y el volumen drásticamente con el aumento de la duración del estrés $y$, este efecto, por consiguiente, aumentó considerablemente la relación parte aérea/raíz.

El periodo de 9 d de anegamiento ocasionó un aumento en el efecto negativo del estrés en el crecimiento de plantas de lulo, como lo corrobora el periodo posterior de recuperación de 9 d que tuvo poco efecto positivo sobre las variables de crecimiento. Así mismo, este periodo de recuperación aumentó el efecto negativo del anegamiento en las variables volumen de raíz y longitud de la raíz en plantas de lulo.

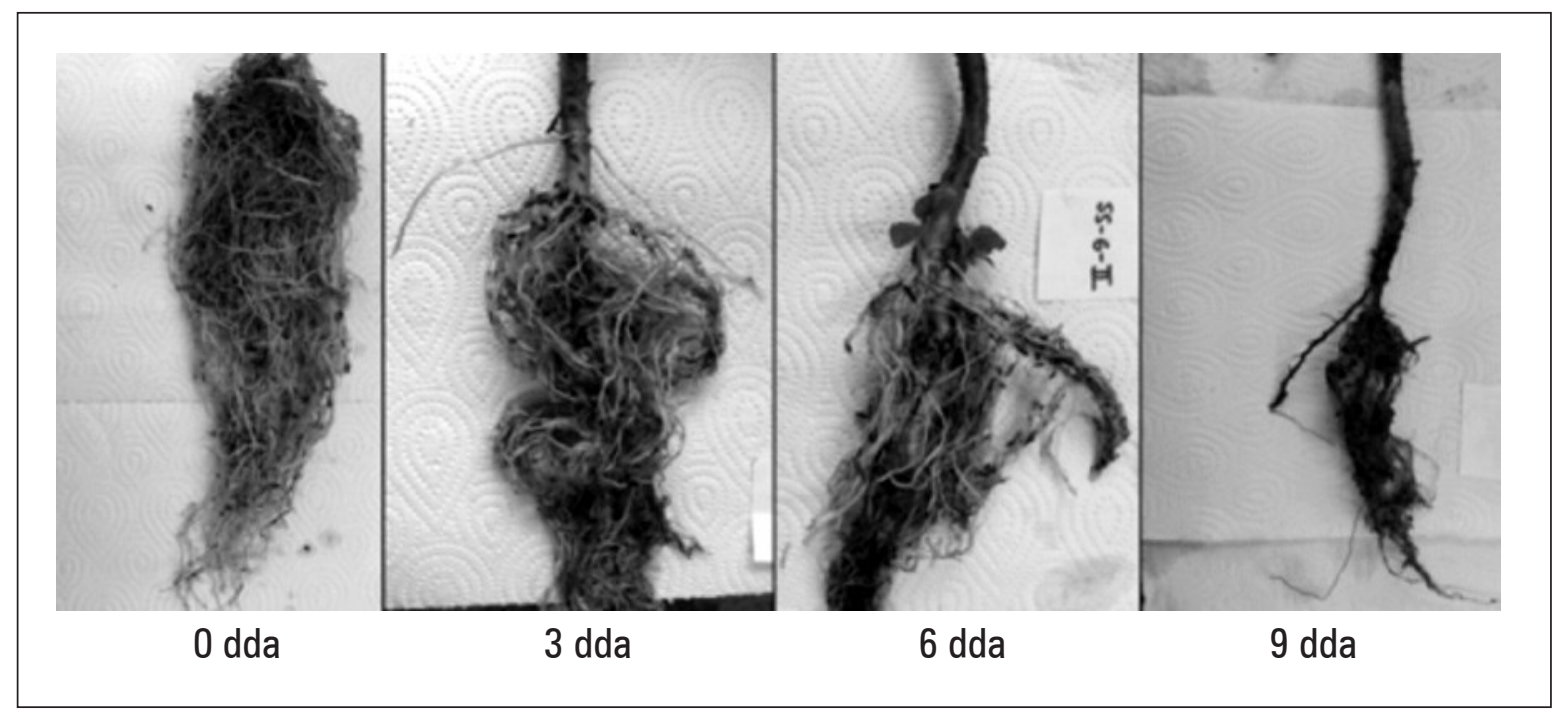

Figura 3. Apariencia de las raíces de plantas de lulo sometidas a cuatro períodos de anegamiento de $0,3,6$ y 9 dda (días de anegamiento) y sin efecto de sombrío (0 dda). 


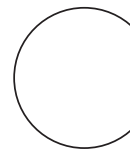

Aldana, F., P. García y G. Fischer. 2014. Effect of waterlogging stress on the growth, development and symptomatology of cape gooseberry (Physalis peruviana L.) plants. Rev. Acad. Colomb. Cienc. 38(149), 393-400. Doi: 10.18257/raccefyn.114.

Bailey-Serres, J., S.C. Lee y E. Brinton. 2012. Waterproofing crops: effective flooding survival strategies. Plant Physiol. 160, 1698-1709. Doi: 10.1104/ pp.112.208173

Bailey-Serres, J. y L. Voesenek. 2008. Flooding stress: Aclimations and genetic diversity. Annu. Rev. Plant Biol. 59, 313-39. Doi: 10.1146/annurev.arplant.59.032607.092752

Baracaldo. A., R. Carvajal, A. Romero, A. Prieto, F. Garcia, G. Fischer y D. Miranda. 2014. El anegamiento afecta el crecimiento y producción de biomasa en tomate chonto (Solanum lycopersicum L.), cultivado bajo sombrío. Rev. Colomb. Cienc. Hortic. 8(1), 92102. Doi: $10.17584 /$ rcch.2014v8i1.2803

Bonet, J. G. y J. F. Cárdenas. 2012. Lulo (Solanum quitoense Lam.). pp. 604-621. En: Fischer, G. (ed.) Manual para el cultivo de frutales en el trópico. Produmedios, Bogotá.

Casierra-Posada, F., K. Pe-a-Olmos, J. Pe-aloza y G. Roveda. 2013. Influencia de la sombra y de micorrizas sobre el crecimiento de plantas de lulo (Solanum quitoense Lam.). Rev. UDCA Act. \&Div. Cient. 16(1), 61-70.

DANE. 2011. Reporte final de áreas afectadas por inundaciones 2010-2011. Departamento Administrativo Nacional de Estadísticas. Bogotá.

Dwivedi, P. y R.S. Dwivedi. 2012. Physiology of abiotic stress in plants. Agrobios, Jodhpur, India.

Fernández, R., R. Perry, J. Flore y R. McLean. 1997. Photosintesis, C-photosynthate distribution and shoot and root growth of young apple trees on 3 rootstocks exposed to flooding. Acta Hortic. 451, 351359. Doi: 10.17660/ActaHortic.1997.451.41

Fischer, G., P.J. Almanza-Merchán y F. Ramírez. 2012. Source-sink relationships in fruit species. A review. Rev. Colomb. Cienc. Hortic. 6(2), 238-253. Doi: 10.17584/rcch.2012v6i2.1980

Fischer, G. y J. Orduz-Rodríguez. 2012. Ecofisiología en frutales. pp. 54-72. En: Fischer, G. (ed.). Manual para el cultivo de frutales en el trópico. Produmedios, Bogotá.

\section{REFERENCIAS BIBLIOGRÁFICAS}

Flórez, S.L., D. Miranda Lasprilla, B. Chaves, G. Fischer y S. Magnitskiy. 2008. Growth of lulo (Solanum quitoense Lam.) plants affected by salinity an substrate. Rev. Bras. Frutic. 30(2), 402-408. Doi: 10.1590/ S0100-29452008000200023

Flórez-Velasco, N., H.E. Balaguera-López y H. RestrepoDíaz. 2015. Effects of foliar urea application on lulo (Solanum quitoense cv. septentrionale) plants grown under different waterlogging and nitrogen conditions. Sci. Hortic. 186, 154-162. Doi: 10.1016/j.scienta.2015.02.021

González, D., L. Ordóñez, P. Vanegas y H. Vásquez. 2014 Cambios en las propiedades fisicoquímicas de frutos de lulo (Solanum quitoense Lam.) cosechados en tres grados de madurez. Acta Agron. 63(1), 11-17. Doi: 10.15446/acag.v63n1.31717

Hansen, P. 1978. Blatt/Frucht-Verhältnisse, Assimilatverteilung und Fruchtentwicklung. Erwerbsobstbau 20, 228-231.

Khondaker, N.A. y K. Ozawa. 2007. Papaya plant growth as affected by soil air oxygen deficiency. Acta Hortic. 740, 225-232. Doi: 10.17660/ActaHortic.2007.740.27

Kozlowski, T.T. 1997. Responses of woody plants to flooding and salinity. Tree Physiol. Monogr. 1, 1-29. Doi: 10.1093/treephys/17.7.490

Laan, P., M. Tosserams, C.W.P.M. Blom y B.W. Veen. 1990. Internal oxygen transport in Rumex species and its significance for respiration under hypoxic conditions. Plant Soil 122, 39-46. Doi: 10.1007/ BF02851908

Lambers, H., F.S. Chapin III y T.L. Pons. 2008. Plant physiological ecology. $2^{\text {nd }}$ ed. Springer, New York, NY. Doi: 10.1007/978-0-387-78341-3

Larcher, W. 2003. Physiological plant ecology. SpringerVerlag, Berlin. Doi: 10.1007/978-3-662-05214-3

Lavinsky. A.O., C.d.S. Sant'Ana, M.S. Mielke, A.A.F de Almeida, F.P. Gomes, S. Franca y D.d.C. Silva. 2007. Effects of light avaibility and soil flooding on growth and photosynthetic characteristics of Genipa americana L. seedlings. New Forests 34, 41-50.

Lobo, M. 2000. Papel de la variabilidad genética en el desarrollo de los frutales andinos como alternativa productiva. pp. 27-36. En: Memorias 3er Seminario de Frutales de Clima Frío Moderado. Corpoica, Manizales, Colombia.

López, M.V. y D.A. del Rosario. 1983. Performance of tomatoes (Lycopersicon lycopersicum (L.) Karsten) un- 
der waterlogged conditions. Philippine J. Crop Sci. 8(2), 75-80.

MADR. 2014. Estadística agropecuaria 2007-2014. Grupo de Estadísticas e Información Sectorial. Ministerio de Agricultura y Desarrollo Rural, Bogotá.

Medina, C.I., E. Martínez, M. Lobo, J.C. López y N. Riao. 2006. Comportamiento bioquímico y del intercambio gaseoso del lulo (Solanum quitoense Lam.) a plena exposición solar en el bosque húmedo montano bajo del oriente antioqueño colombiano. Rev. Fac. Nac. Agron. Medellín 59(1), 3123-3146.

Messinger, J. y M. Lauerer. 2015. Solanum quitoense, a new greenhouse crop for Central Europe: Flowering and fruiting respond to photoperiod. Sci. Hortic. 183, 23-30. Doi: 10.1016/j.scienta.2014.11.015

Mielke, M.S., E.M. Matos, V.B. Couto, A.-A.F. de Almeida, F.P. Gomes y P.A.O. Mangabeira. 2005. Some photosynthetic and growth responses of Annona glabra L. seedlings to soil flooding. Acta Bot. Bras. 19, 905-911. Doi: 10.1590/S010233062005000400025

Mielke, M.S. y B. Schaffer. 2010. Photosynthetic and growth responses of Eugenia uniflora L. seedlings to soil flooding and light intensity. Environ. Exp. Bot. 68,113-121. Doi: 10.1016/j.envexpbot.2009.11.007

Mommer, L., H. de Kroon, R. Pierik, G.M. Bögemann y E.J.W. Visser. 2005. A functional comparison of acclimation to shade and submergence in two terrestrial plant species. New Phytologist 167, 197-206. Doi: 10.1111/j.1469-8137.2005.01404.x

Moreno, A. y G. Fischer. 2014. Efectos del anegamiento en los frutales. Una revisión. Temas Agrarios 19(1), 108-125.

Morton, J. 1987. Naranjilla (Solanum quitoense Lam., Solanum angulatum Lam.). pp. 425-428. En: Dowling, C.F. (ed.). Fruits of warm climates. Creative Resources Systems, Inc., Miama, FL.

Najeeb, U., M.B. Bange, D.K.Y. Tan y B.J. Atwell. 2015. Consequences of waterlogging in cotton and opportunities for mitigation of yield losses. AoB Plants 7. Doi: 10.1093/aobpla/plv080

Núñez-Elisea, R., B. Schaffer, J.B. Fisher, A.M. Colls y J.H. Crane. 1999. Influence of flooding on net CO2 assimilation, growth and stem anatomy of $\mathrm{An}$ nona species. Ann. Bot. 84, 771-780. Doi: 10.1006/ anbo.1999.0977

Pallardy, S.G. 2007. Physiology of woody plants. $3^{\text {a }}$ ed. Academic Press, San Diego, CA.

Pardos. J. 2004. Respuestas de las plantas al anegamiento del suelo. Invest. Agrar: Sist. Recur. For. (Fuera de serie), 101-107.
Sairam, R.K., D. Kumutha, K. Ezhilmathi, P.S. Deshmukh y G.C. Srivastava. 2008. Physiology and biochemistry of waterlogging tolerance in plants. Biol. Plant. 52(3), 401-412. Doi: 10.1007/s10535-008-0084-6

Sauter, M. 2013. Root response to flooding. Curr. Opinion Plant Biol. 16, 282-286. Doi: 10.1016/j. pbi.2013.03.013

Steffens, D., B.W. Hütsch, T. Eschholz, T. Lošáky S. Schubert. 2005. Water logging may inhibit plant growth primarily by nutrient deficiency rather than nutrient toxicity. Plant Soil Environ. 51(12), 545-552.

Striker, G.G. 2012. Flooding stress on plants: anatomical, morphological and phys-iological responses. pp. 3-28. In: Botany. InTech, Rijeka, Croacia.

Taiz, L. y E. Zeiger. 2010. Plant physiology. 3a ed. Sinauer Associates, Sunderland, MA.

Torres, C., G. Fischer y D. Miranda. 2016. Principales fisiopatías del cultivo de uchuva (Physalis peruviana L.). pp. 139-146. En: Miranda, D., C. Carranza y G. Fischer (eds.). Problemas de campo asociados al cultivo de uchuva (Physalis peruviana L.). Editorial Universidad Nacional de Colombia, Bogotá.

Valladares, F. y U. Niinemets. 2008. Partial sunlight tolerance, a key plant feature of complex nature and consequences. Annu. Rev. Ecol. Evol. Syst. 39, 237257. Doi: 10.1146/annurev.ecolsys.39.110707.173506

Vartapetian, B.B. y M.B. Jackson. 1997. Plant adaptations to anaerobic stress. Ann. Bot. 79, 3-20. Doi: 10.1093/oxfordjournals.aob.a010303

Vidoz, M.L., F. Mignolli, H.T. Aispuru, L.A. Mroginski. 2016. Rapid formation of adventitious roots and partial ethylene sensitivity result in faster adaptation to flooding in the aerial roots (aer) mutant of tomato. Sci. Hortic. 201, 130-139. Doi: 10.1016/j. scienta.2016.01.032

Zeng, F., L. Shabala, M. Zhou, G. Zhang y S. Shabala. 2013. Barley responses to combined waterlogging and salinity stress: separating effects of oxygen deprivation and elemental toxicity. Frontiers Plant Sci. 4(313). Doi: 10.1093/treephys/tpv089

Yamauchi, T., S. Shimamurab, M. Nakazonoa y T. Mochizukic. 2013. Aerenchyma formation in crop species: A review. Field Crops Res. 152, 8-16. Doi: 10.1016/j.fcr.2012.12.008

Yetisir, H., M.E. Caliskan, S. Soylu y M. Saka. 2006. Some physiological and growth responses of watermelon [Citrullus lanatus (Thunb.) Matsum. and Nakai] grafted onto Lagenaria siceraria to flooding. Environ. Exp. Bot. 58, 1-8. Doi: 10.1016/j.envexpbot.2005.06.010 